\title{
Mesomelic dysplasia Kantaputra type is associated with duplications of the HOXD locus on chromosome $2 q$
}

\author{
Piranit N Kantaputra ${ }^{1,9}$, Eva Klopocki ${ }^{2,3,9}$, Bianca P Hennig ${ }^{2,3}$, Verayuth Praphanphoj ${ }^{4}$, Cédric Le Caignec ${ }^{5,6}$, \\ Bertrand Isidor $^{5}$, Mei L Kwee ${ }^{7}$, Deborah J Shears ${ }^{8}$ and Stefan Mundlos ${ }^{\star, 2,3}$

\begin{abstract}
Mesomelic dysplasia Kantaputra type (MDK) is characterized by marked mesomelic shortening of the upper and lower limbs originally described in a Thai family. To identify the cause of MDK, we performed array CGH and identified two microduplications on chromosome 2 (2q31.1-q31.2) encompassing $\sim 481$ and $507 \mathrm{~kb}$, separated by a segment of normal copy number. The more centromeric duplication encompasses the entire HOXD cluster, as well as the neighboring genes EVX2 and MTX2. The breakpoints of the duplication localize to the same region as the previously identified inversion of the mouse mutant ulnaless (UI), which has a similar phenotype as MDK. We propose that MDK is caused by duplications that modify
\end{abstract} \\ the topography of the locus and as such result in deregulation of HOXD gene expression.
}

European Journal of Human Genetics (2010) 18, 1310-1314; doi:10.1038/ejhg.2010.116; published online 21 July 2010

Keywords: HOXD; MDK; microduplication

\section{INTRODUCTION}

Mesomelic dysplasia Kantaputra type (MDK, MIM 156232) is a rare dominantly inherited skeletal disease characterized by symmetric marked shortening of the upper and lower limbs. ${ }^{1,2}$ The ulnae are very short, and the radii are bowed. The distal humerus has a dumbbell shape, the hands are relatively normal but show progressive flexion contractures of the proximal interphalangeal joints. Carpal and tarsal synostoses are observed in some individuals. In the lower limbs, feet are fixed in plantar flexion with sole facing backward, causing 'ballerina-like standing. The prominent distal fibula on the ventral aspect is a consistent feature and considered as the 'signature' finding of the syndrome. Calcaneus is small or missing. Small fibula and talus, and fibulo-calcaneal synostosis are characteristic features. Tibial bony knot articulates with the proximal end of the fibula. Since the original description, several other cases have been described that show similar changes. ${ }^{3,4}$ In an Italian family with a mesomelic dysplasia, a balanced translocation $\mathrm{t}(2 ; 8)$ ( $\mathrm{q} 31 ; \mathrm{p} 21)$ had previously been observed. ${ }^{5,6}$ On the basis of the phenotypic similarities with MDK, chromosomes 2 and 8 were tested as candidate regions and a region on chromosome 2 (2q24-q32) spanning about $22.7 \mathrm{cM}$ was identified to harbor the MDK gene. ${ }^{7}$ Interestingly, this interval contains the HOXD cluster. However, sequencing of individual genes of the HOXD cluster revealed no mutation associated with MDK. We here describe genomic rearrangements including the entire HOXD cluster identified in the Thai family originally described by Kantaputra et $a l^{1,2}$ as the likely cause for MDK.

\section{MATERIALS AND METHODS}

Genomic DNA samples were obtained from EDTA blood. DNA of the nine living affected individuals and one unaffected individual (IV.23) from pedigree of the publication of Kantaputra et $a l^{2}$ were available for genomic analysis by microarray-based comparative genomic hybridization (array CGH) and quantitative real-time PCR (qPCR). In addition, we obtained DNA samples of the MDK-affected individual II.1 from the publication of Shears $e t a l^{3}$ and II.1 from the publication of Kwee et al. ${ }^{4}$

\section{Microarray-based comparative genomic hybridization}

DNA of the affected individual III.26, ${ }^{2}$ II. $1^{3}$ and II. $1^{4}$ were available for array-CGH analysis. Array CGH was performed using a whole-genome oligonucleotide array (244K, Agilent Technologies, Santa Clara, CA, USA). $244 \mathrm{~K}$ image data were analyzed using Feature Extraction 9.5.3.1 and CGH Analytics 3.4.40 software (Agilent Technologies) with the following analysis settings: aberration algorithm ADM-2; threshold: 6.0 ; window size: $0.2 \mathrm{Mb}$; filter: 5 probes, $\log 2$ ratio $=0.29$. The hybridization on a custom tiling array (AMADID no. 023120; Agilent Technologies), which covers $3 \mathrm{Mb}$ of the critical region at high density allowed a more precise determination of the duplication size and breakpoint positions and was performed exclusively with the Thai family member III.26. ${ }^{2}$ All genomic positions given in the text are according to human genome version hg18 (UCSC Genome browser, Mar. 2006; http:// www.genome.ucsc.edu/).

\section{Quantitative real-time PCR}

Confirmation of the array-CGH result of individual III. $26^{2}$ and analysis of further nine members (eight affected, one unaffected) of the Thai MDK family ${ }^{2}$ to show the segregation was carried out by qPCR. Amplicons P1 and P3 are flanking the centromeric duplication and amplicons P4 and P6 are flanking the telomeric duplication. Amplicons P2 and P5 are located within the centromeric and telomeric duplication, respectively. Primer sequences can be obtained on request.

qPCR was performed on ABI Prism 7900HT Sequence Detection System (Applied Biosystems, Foster City, CA, USA) in a total volume of $12 \mu \mathrm{l}$ in each well containing $6 \mu$ l of SYBR-Green PCR Master Mix (ABI SYBR Green PCR Master Mix, Applied Biosystems), $10 \mathrm{ng}$ of genomic DNA (10 $\mu \mathrm{l})$ and $1 \mu \mathrm{l}$

${ }^{1}$ Department of Pediatric Dentistry, Craniofacial Genetics Laboratory, Faculty of Dentistry, Chiang Mai University, Chiang Mai, Thailand; ${ }^{2}$ Institut für Medizinische Genetik, Charité Universitätsmedizin Berlin, Berlin, Germany; ${ }^{3}$ Max-Planck-Institut für Molekulare Genetik, Berlin, Germany; ${ }^{4}$ Genetic Laboratory Rajanukul Institute, Bangkok, Thailand; ${ }^{5}$ Service de Génétique Médicale, Centre Hospitalier Universitaire de Nantes, Nantes, France; ${ }^{6}$ INSERM, UMR915, I'institut du thorax, Nantes, France; ${ }^{7}$ Department of Clinical Genetics, VU University Medical Centre, Amsterdam, The Netherlands; ${ }^{8}$ Clinical and Molecular Genetics Unit, Institute of Child Health, London, UK

*Correspondence: Dr S Mundlos, Institut für Medizinische Genetik, Charité Universitätsmedizin Berlin, Augustenburger Platz 1, 13353 Berlin, Germany.

Tel: +49 30450569 122; Fax: +49 30450569 915; E-mail: stefan.mundlos@charite.de

${ }^{9}$ These authors contributed equally to this work.

Received 20 January 2010; revised 25 May 2010; accepted 9 June 2010; published online 21 July 2010 
primers $(0.2 \mu$ mol each). Samples were run in triplicates in separate tubes to permit the quantification of the target sequences normalized to albumin (ALB). PCR conditions were according to manufacturer's protocol: initial denaturation step at $95^{\circ} \mathrm{C}$ for $8 \mathrm{~min}$ followed by 40 cycles with denaturation at $95^{\circ} \mathrm{C}$ for $15 \mathrm{~s}$ and a combined annealing/elongation step at $60^{\circ} \mathrm{C}$ for $1 \mathrm{~min}$. Using calibrator samples of normal control genomic DNA, the gene copy number was estimated based on the ddCt method. In addition, we performed an identification of the individuals' genders by calculating the coagulation factor VIII (F8, Xq28) relative to the two-copy-control ALB to assure its reliability.

\section{Fluorescence in situ hybridization}

BAC clones RP4-745K6 and RP11-279N12 (obtained from the imaGenes, Berlin, Germany) were fluorescently labeled using nick translation, and hybridized to metaphase spreads of the individual III.11's lymphocytes using standard procedures. BAC clones were labeled with Spectrum Orange (RP4-745K6, located in centromeric duplication; Vysis Inc., Downers Grove, IL, USA) and Spectrum Green (RP11-279N12, located in telomeric duplication; Vysis Inc.). Chromosomes were counterstained with DAPI. Image analysis was performed using the ISIS analysis system (Metasystems, Altlussheim, Germany)

\section{RESULTS}

As submicroscopic aberrations are a known cause for congenital limb malformations, ${ }^{8-10}$ we performed array CGH using a wholegenome oligonucleotide array $(244 \mathrm{~K}$, Agilent Technologies) of one affected individual (III. 26) from the MDK family initially described by Kantaputra. ${ }^{2}$ Using this technology, we identified two microduplications on chromosome 2 (2q31.1-q31.2) encompassing $\sim 481$ and $507 \mathrm{~kb}$, respectively, separated by a segment of normal copy number. The centromeric duplication contained the entire HOXD locus plus EVX2 and MTX2, whereas the telomeric duplication contained the genes NEF2L2 and HNRNPA3. To determine the duplication sizes and the breakpoints in more detail, we designed a custom tiling array of the entire region of $\sim 3 \mathrm{Mb}$. This technology confirmed both duplications in the affected MDK family member III.26 $6^{2}$ and positioned them at 176506567-176980136 (centromeric duplication) and 177342561-177854004 (telomeric duplication), respectively (Figure 1a). A cryptic translocation or insertion of the duplicated regions into another chromosome was excluded by fluorescence in situ hybridization analysis (data not shown).

Using qPCR analyses, we were able to confirm the duplications and further narrow down their boundaries: (1) centromeric duplication, proximal breakpoint between 176506582 and 176507065 and distal breakpoint between 176985080 and 176986 597; (2) telomeric duplication, proximal breakpoint between 177342108 and 177342602 and distal breakpoint between 177856721 and 177857706 (data not shown). The proximal breakpoint of the centromeric duplication was thus located within the gene KIAA1715 and the distal breakpoint $\sim 221 \mathrm{~kb}$ distal of MTX2. The telomeric duplication started $\sim 357.5 \mathrm{~kb}$ away from the centromeric duplication and ended in the noncoding region between the genes NFE2L2 and APGS. In addition, we identified a small amplification at the distal end of the centromeric duplication (chr2:176 973 781-176980 910) (Figure 1a). However, this region overlaps with a benign copy-number variation
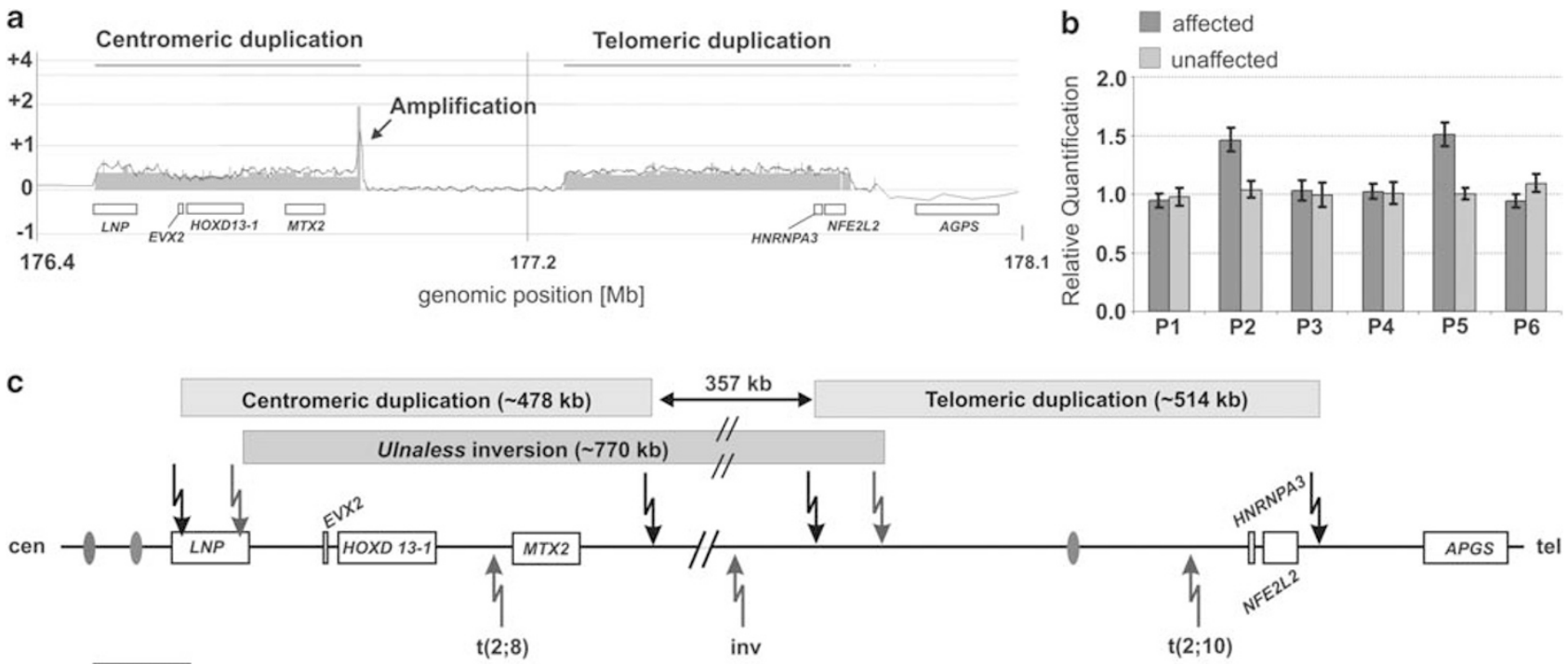

$100 \mathrm{~kb}$

Figure 1 (a) Profile of custom tiling array-CGH analysis, which covers $3 \mathrm{Mb}$ of the critical region on chromosome $2 \mathrm{q}$ at high density demonstrating two duplications separated by a segment of normal copy number in individual III.26. ${ }^{2}$ The centromeric duplication encompasses the HOXD cluster. A small amplification at the distal end of the centromeric duplication is indicated. Regions with a log 2 ratio $>0.3$ represent duplications and are shaded in gray. Genomic positions are according to human genome version hg18. (b) Microduplications and segregation confirmed by qPCR. For one affected (individual III. $16 ;^{2}$ dark gray bars) and one unaffected (individual IV. $23 ;^{2}$ light gray bars) individual, mean values and SDs (error bars) for each target amplicon relative to Albumin as a two-copy reference gene were calculated. The amplicons P2 and P4 located within the centromeric and telomeric duplicated region, respectively, show a ratio of $\sim 1.5$ in the affected individual indicating a copy-number gain, whereas the unaffected individual shows a ratio of $\sim 1.0$, which corresponds to a normal copy number (two copies). The unaffected individual is the daughter of the affected individual III.26 with whom we performed the array $\mathrm{CGH}$ shown in (a). (c) Schematic illustration of aberrations at the HOXD cluster and flanking region on chromosome $2 \mathrm{q}$ associated with limb malformations. The two duplications detected in the MDK family (gray bars), as well as the inversion in the mouse mutant ulnaless (green bar) are indicated. The centromere (cen) is on the left, and telomere (tel) on the right. The breakpoints are indicated by arrows in the corresponding colors. Red arrows below indicate published translocations and the inversion (inv). ${ }^{6,17}$ The translocation $t(2 ; 8)$ and the inversion result in mesomelic shortening. Regulatory elements are indicated by colored ovals (blue: global control region (GCR); red: centromeric repressor (CenR); green: early limb control region (ELCR)). Genomic positions are according to human genome version hg18. The color reproduction of the figure is available on the html full text version of the paper. 
(chr2:176973 862-176980 178) described by Kim et al ${ }^{11}$ and it is therefore most likely not clinically relevant. Segregation of the duplications with the MDK phenotype was confirmed by qPCR analysis of further nine family members from the Thai family (eight affected, one unaffected). Amplicons P2 and P5, which localize within the duplications, detected a normal copy number in the unaffected individual IV.23 (Figure 1b). The unaffected individual is the daughter of the affected female III.26 tested by array CGH. To investigate other nonrelated individuals with MDK, we tested two previously published cases by array CGH. ${ }^{3,4}$ We were not able to identify a clinically relevant copy-number change, not within the region on chromosome $2 \mathrm{q}$, nor elsewhere in the genome, indicating that MDK is a genetically heterogeneous condition.

\section{DISCUSSION}

Homeobox genes, and the proteins they encode, the homeodomain proteins, have important roles in the developmental processes of many multicellular organisms. In mammals, four such clusters exist that harbor a total of 49 genes. During development of the limbs, Hox genes of the $A$ and $D$ clusters, and in particular those of the 5 ' end, that is, Hoxa10, - $a 11,-a 13$, Hoxd9, $-d 10,-d 11,-d 12,-d 13$, are of particular importance. ${ }^{12}$ Overall they appear to systematically involve loss or reduction of skeletal elements, and their function has accordingly been interpreted to specify individual segments of the limb in a proximo-distal manner.

In humans, mutations in HOX genes results in a number of limb malformations including synpolydactyly (HOXD13), and hand-foot-genital syndrome (HOXA13). ${ }^{13}$ Larger deletions of the entire HOXD cluster and a smaller deletion removing just the HOXD9-13 and EVX2 genes were reported to result in mild limb malformations, including fifth-finger clinodactyly, variable cutaneous syndactyly of the toes, hypoplastic middle phalanges of the feet and synpolydactyly. ${ }^{14,15}$ This phenotype is similar to the mild synpolydactyly phenotype commonly found in carriers of specific HOXD13 mutations, indicating that even deletions of the entire HOXD cluster need not to produce major limb defects. ${ }^{13}$

Several chromosomal translocations located within the vicinity of the HOXD cluster have been described that result in severe phenotypes (Figure 1). Two rearrangement breakpoints located on the telomeric side of the HOXD cluster were described to result in proximal malformations of the limbs. ${ }^{16}$ In one individual, a symmetrical limb phenotype consisting of ulnar aplasia, radial shortening, and absence of third to fifth rays was described in association with the translocation $\mathrm{t}(2 ; 10)(\mathrm{q} 31.1 ; \mathrm{q} 23.33)$. The breakpoint was localized $\sim 950 \mathrm{~kb}$ away from the $3^{\prime}$ end of the Hoxd cluster. In the other case, a paracentric inversion $(\operatorname{inv}(2)(\mathrm{p} 15 \mathrm{q} 31))$ was associated with hypoplastic ulnae, radii and fibulae and small tibiae. This phenotype is somewhat reminiscent of the changes seen in MDK. The breakpoint in this case was closer to the cluster with a distance of $\sim 500 \mathrm{~kb}$ from Hoxd1. ${ }^{16}$

Furthermore, a refinement of the translocation $\mathrm{t}(2 ; 8)$ of the patient with mesomelic shortening originally described by Ventruto et al ${ }^{5}$ located this breakpoint $56 \mathrm{~kb}$ away from the $3^{\prime}$ end of the Hoxd cluster $^{6,17}$ (for overview see Figure 1c).

The above-described breakpoints localize within or close to the duplications described here. Several other genes besides the HOXD cluster are present in this region that are potential candidates for MDK. The centromeric duplication contains parts of KIAA1715, EVX2 and MTX2. KIAA1715, also known as Lunapark (LNP), is expressed in the developing digits but does not seem to have an important function during digit development. ${ }^{18} \mathrm{EVX} 2$, located next to
Hoxd13, is also expressed in the digits. Inactivation of Evx2 in the mouse results in weak skeletal alterations of the digits. ${ }^{19}$ An additional copy of EVX2 may thus have an effect on limb development, but the main effect of Evx2 appears to be in the autopod, the region of the limb that shows little changes in MDK. MTX2 (MIM 608555) encodes for Metataxin, a protein that localizes to the outer mitochondrial membrane. It is not expressed in the developing $\operatorname{limb}^{17}$ and as such unlikely to contribute to the phenotype. The telomeric duplication neighbors the AGPS gene, a member of the FAD-binding oxidoreductase/transferase type 4 family involved in long-chain fatty acid metabolism. Mutations in this gene have been associated with rhizomelic chondrodysplasia punctata, type 3 (MIM 600121) and Zellweger syndrome (MIM 214100). Located within the duplicated region are HNRNPA3 and NFE2L2 (MIM 600492). The latter encodes a transcription activator that is important for the coordinated upregulation of genes in response to oxidative stress. HNRNPA3 has a role in cytoplasmic trafficking of RNA and may be involved in pre-mRNA splicing. In summary, it is unlikely that either of these genes is involved in the pathogenesis of MDK.

Several lines of evidence suggest, however, that HOXD genes are the most likely candidates for the MDK phenotype. Hox genes show specific proximo-distal expression patterns that specify the future skeletal elements. Hox $d 9$ and $-d 10$, for example, are expressed in the stylopod, the future humerus, Hoxd11 and Hoxal1 in the zeugopod, the future radius/ulna, and Hoxd13, Hoxd12 and Hoxa13 in distal limb (autopod). Inactivation of paralogous genes results in severe shortening of the corresponding elements. A knockout of Hoxd11 and Hoxa11, for example, results in a severe reduction deficiency of radius and ulna, similar to the MDK phenotype. ${ }^{20,21}$ MDK-like malformations are also present in the mouse mutant ulnaless $(U l)$, which is a semidominant X-ray-induced mutant with very short zeugopods with an almost complete absence of the ulna. Gene expression studies have shown that $U l$ embryos show an ectopic expression of Hoxd13 and Hoxd12 in the proximal limb (zeugopod) and a reduction of Hoxd13, Hoxd12 and Hoxd11 in the autopod. ${ }^{22,23}$ The skeletal reductions of the $U l$ mutant were interpreted as a consequence of posterior prevalence, whereby proximal misexpression of Hoxd13 and Hoxd12 results in the transcriptional and/or functional inactivation of the more anterior, that is, Hox group 11 genes. The hypothesis of misregulated Hoxd13 expression was supported by the finding that $U l$ was caused by a balanced paracentric inversion of $\sim 770 \mathrm{~kb}$ in size, with one breakpoint centromeric to the Hoxd cluster disrupting the Lnp gene, and the other breakpoint telomeric to the Hoxd cluster ${ }^{18}$ (Figure 1b). The inversion included Evx2, the Hoxd locus and Mtx2, and is thus very similar to the centromeric duplication identified in the MDK family.

The pathogenesis of $U l$ was further clarified by an elegant series of experiments that led to the identification of a Hoxd control region located at the $5^{\prime}$ (centromeric) side $\sim 200 \mathrm{~kb}$ away from the $5^{\prime}$ end of the Hoxd cluster. ${ }^{18}$ This so-called global control region (GCR, blue oval, Figures 1c and 2) positively influences the expression of Evx2 and other $5^{\prime}$ Hoxd genes expressed in the distal limb and suppresses their expression in the proximal limb. Recently, Tarchini and Duboule ${ }^{25}$ specified an additional regulatory element centromeric to the HOXD cluster, which controls the posterior restriction of $5^{\prime}$ Hoxd genes (CenR, red oval, Figures 1c and 2). These authors claimed that the GCR preferentially controls the expression in the presumptive digit domain. In the case of the $U l$ inversion, the centromeric breakpoint is located between the GCR, the additional centromeric regulator, and the cluster thus separating the cluster from the regulatory influence of 
a wild type

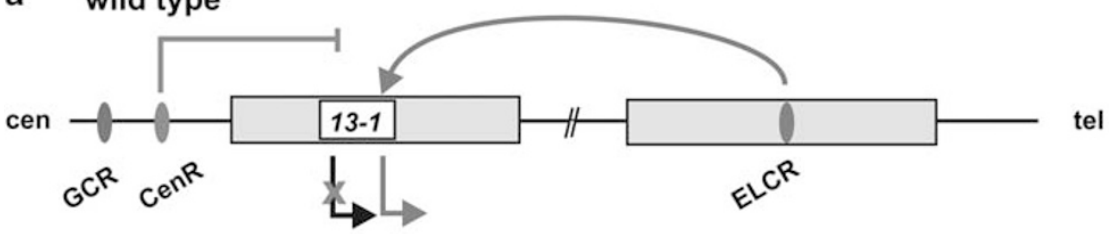

b MDK associated duplications

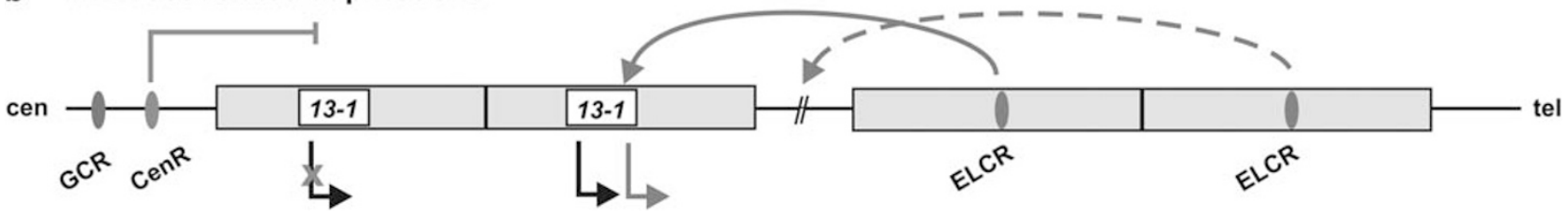

Figure 2 (a) Regulation of HOXD cluster during early limb development. A complex time-dependent mechanism involving two control elements on the centromeric and telomeric side of the HOXD cluster controls the expression of HOXD genes in the early limb bud. One regulatory element centromeric of the HOXD cluster (CenR, red oval) represses the expression of 5' HOXD genes (HOXD10-13) indicated by the red bar. ${ }^{25}$ The transcriptional activation of the $3^{\prime}$ HOXD genes (HOXD1-9) is simultaneously induced by the early limb control region (ELCR, green oval), which is located on the telomeric side of the HOXD cluster indicated by the green arrow ${ }^{24}$ (Tarchini and Duboule, 2006). Small green arrow marks active genes. The centromere (cen) is on the left, and telomere (tel) on the right. GCR, global control region; CenR, centromeric repressor. (b) Hypothesis on disturbance of HOXD cluster regulation in MDK. The two duplications detected in the MDK family (gray bars) are arranged in direct tandem orientation and result in a duplication of the HOXD cluster (centromeric dup) and of the ELCR (telomeric dup). As the ELCR is promoter independent, the onset of transcription depends on the distance between gene and ELCR. The duplicated HOXD cluster can be activated by the ELCR (small arrows); however, the 5' HOXD genes (HOXD10-13) escape the repression by the centromeric regulator CenR and thus the posterior restriction. As a consequence, HOXD13 is expressed too early and ectopically leading to the MDK phenotype. The later HOXD expression in the digit domain controlled by the GCR is unaffected by the genomic duplications. The color reproduction of the figure is available on the $\mathrm{html}$ full text version of the paper.

the GCR and the additional centromeric regulator. The authors suggested that the disconnection of $5^{\prime}$ Hoxd genes from the regulator results in a downregulation of $5^{\prime}$ Hoxd genes in the distal limb (autopod) and an upregulation in the proximal limb. ${ }^{25}$ The duplication described here may have a similar effect. Similar to the $U l$ inversion, the duplicated cluster would be located more than $500 \mathrm{~kb}$ away from the GCR and the additional centromeric regulator thus escaping its control (Figure $2 \mathrm{~b}$ ).

The presence of two breakpoints at the telomeric side of the cluster that result in an MDK-like phenotype suggests an alternative explanation in which the responsible regulator is located $3^{\prime}$ of HOXD1. Indeed, Zakany et a ${ }^{24}$ propose the presence of an early limb control region (ELCR, green oval, Figures $1 \mathrm{c}$ and 2 ) localized telomeric to the cluster, which is needed to implement colinear expression of the HOXD cluster. The exact position of the ELCR is not known. One of the duplications detected in the Thai family is expected to disconnect the $5^{\prime}$-located cluster from the $3^{\prime}$-located regulator and vice versa. As a consequence, HOXD13 is expressed too early and ectopically leading to the MDK phenotype (Figure $2 \mathrm{~b}$ ). Thus, it is possible that one or both regulators have to be affected to produce the MDK/Ul phenotype. To verify our results of the Thai family, we tested the further described nonrelated MDK individuals. ${ }^{3,4}$ We were not able to identify a clinically relevant copy-number change anywhere in the genome of these two additional individuals. However, as the array-CGH analysis does not exclude balanced structural rearrangements, one can speculate that the MDK phenotype in patients reported by Shears et al and Kwee et $a l^{3,4}$ and the Thai patients reported in this paper is caused by chromosomal rearrangements affecting HOXD locus regulation. The identification of further rearrangements in this region will help to clarify this.

\section{CONFLICT OF INTEREST}

The authors declare no conflict of interest.

\section{ACKNOWLEDGEMENTS}

We thank the family for participating in this study. This work was supported by grants from the Deutsche Forschungsgemeinschaft to SM and EK, and the Thailand Research Fund (TRF) to PK.

1 Kantaputra PN, Gorlin RJ, Langer Jr LO: Dominant mesomelic dysplasia, ankle, carpal, and tarsal synostosis type: a new autosomal dominant bone disorder. Am J Med Genet 1992; 44: 730-737.

2 Kantaputra PN: Thirteen-year-follow up report on mesomelic dysplasia, Kantaputra type (MDK), and comments on the paper of the second reported family of MDK by Shears et al. Am J Med Genet A 2004; 128A: 1-5.

3 Shears DJ, Offiah A, Rutland P, Sirimanna T, Bitner-Glindzicz M, Hall C: Kantaputra mesomelic dysplasia: a second reported family. Am J Med Genet A 2004; 128A: $6-11$.

4 Kwee ML, van de Sluijs JA, van Vugt JM, Wijnaendts LC, Gille JJ: Mesomelic dysplasia, Kantaputra type: clinical report, prenatal diagnosis, no evidence for SHOX deletion/ mutation. Am J Med Genet A 2004; 128A: 404-409.

5 Ventruto V, Festa B, Renda S et al: [Phenotype anomalies in subjects with balanced chromosome translocation. Presentation of 4 cases]. Pathologica 1983; 75 (Suppl): 258-261.

6 Sugawara H, Egashira M, Harada $\mathrm{N}$ et al: Breakpoint analysis of a familial balanced translocation $\mathrm{t}(2 ; 8)(\mathrm{q} 31 ; \mathrm{p} 21)$ associated with mesomelic dysplasia. J Med Genet 2002; 39: E34.

7 Fujimoto M, Kantaputra PN, Ikegawa $S$ et al: The gene for mesomelic dysplasia Kantaputra type is mapped to chromosome 2q24-q32. J Hum Genet 1998; 43: 32-36.

8 Klopocki E, Schulze H, Strauss G et al: Complex inheritance pattern resembling autosomal recessive inheritance involving a microdeletion in thrombocytopenia-absent radius syndrome. Am J Hum Genet 2007; 80: 232-240.

9 Klopocki E, Ott CE, Benatar N, Ullmann R, Mundlos S, Lehmann K: A microduplication of the long range SHH limb regulator (ZRS) is associated with triphalangeal thumbpolysyndactyly syndrome. J Med Genet 2008; 45: 370-375.

10 Dathe K, Kjaer KW, Brehm A et al: Duplications involving a conserved regulatory element downstream of BMP2 are associated with brachydydactyly type A2. Am J Hum Genet 2009; 84: 483-492.

$11 \mathrm{Kim} \mathrm{JI}$, Ju YS, Park $\mathrm{H}$ et al: A highly annotated whole-genome sequence of a Korean individual. Nature 2009; 460: 1011-1015.

12 Zakany J, Duboule D: The role of Hox genes during vertebrate limb development. Curr Opin Genet Dev 2007; 17: 359-366.

13 Goodman FR: Limb malformations and the human HOX genes. Am J Med Genet 2002; 112: 256-265. 
14 Prieur M, Lapierre J, Le Lorch M et al: HOXD gene cluster haploinsufficiency does not generate gross limb abnormalities. Eur J Hum Genet 2000; 8 (Suppl. 1): 74.

15 Slavotinek A, Schwarz C, Getty JF, Stecko O, Goodman F, Kingston H: Two cases with interstitial deletions of chromosome 2 and sex reversal in one. Am J Med Genet 1999; 86: 75-81.

16 Dlugaszewska B, Silahtaroglu A, Menzel C et al: Breakpoints around the HOXD cluster result in various limb malformations. J Med Genet 2006; 43: 111-118.

17 Spitz F, Montavon T, Monso-Hinard C et al: A t $(2 ; 8)$ balanced translocation with breakpoints near the human HOXD complex causes mesomelic dysplasia and vertebral defects. Genomics 2002; 79: 493-498.

18 Spitz F, Gonzalez F, Duboule D: A global control region defines a chromosomal regulatory landscape containing the HoxD cluster. Cell 2003; 113: 405-417.

19 Herault Y, Hraba-Renevey S, van der Hoeven F, Duboule D: Function of the Evx-2 gene in the morphogenesis of vertebrate limbs. EMBO J 1996; 15: 6727-6738.
20 Wellik DM, Capecchi MR: Hox10 and Hox11 genes are required to globally pattern the mammalian skeleton. Science 2003; 301: 363-367.

21 Boulet AM, Capecchi MR: Multiple roles of Hoxa11 and Hoxd11 in the formation of the mammalian forelimb zeugopod. Development 2004; 131: 299-309.

22 Peichel CL, Prabhakaran B, Vogt TF: The mouse Ulnaless mutation deregulates posterior HoxD gene expression and alters appendicular patterning. Development 1997; 124: 3481-3492.

23 Herault Y, Fraudeau N, Zakany J, Duboule D: Ulnaless (UI), a regulatory mutation inducing both loss-of-function and gain-of-function of posterior Hoxd genes. Development 1997; 124: 3493-3500.

24 Zakany J, Kmita M, Duboule D: A dual role for Hox genes in limb anterior-posterior asymmetry. Science 2004; 304: 1669-1672.

25 Tarchini B, Duboule D: Control of Hoxd genes' collinearity during early limb development. Dev Cell 2006; 10: 93-103. 